http://jmscr.igmpublication.org/home/ ISSN (e)-2347-176x ISSN (p) 2455-0450 crossref DOI: https://dx.doi.org/10.18535/jmscr/v8i11.82

\title{
Study of Effects of Acute Myocardial Infarction on Cholesterol and Cholesterol Ratios
}

\author{
Authors \\ Dr Yarraguntla Pranathi ${ }^{1}$, Dr D. Radha Krishnan ${ }^{2}$, Dr V.Navya $\mathrm{Sri}^{3}$ \\ ${ }^{1}$ Post graduate, Department of General Medicine \\ ${ }^{2}$ Professor, Department of General Medicine \\ ${ }^{3}$ Post graduate, Department of General Medicine
}

\begin{abstract}
Background: Coronary artery disease remains the most common cause of death despite significant advancement in its prevention and treatment ${ }^{3}$. Myocardial infarction is facilitated by many risk factors, of which dyslipide MI $a$ is an important factor. In patients with acute myocardial infarction cholesterol levels are no longer valid after $24 \mathrm{hrs}$ from presentation because acute MI causes rapid decline in serum levels of total cholesterol and triglycerides.

Materials and Methods: This is a cross sectional study conducted at the intensive care unit and medical wards of King George hospital, Visakhapatnam from January 2020 to august 2020. A total of 100 patients with confirmed diagnosis of acute myocardial infarction enrolled in the study. Serum levels of total cholesterol, HDL cholesterol, LDL cholesterol, triglycerides on day- 1 post MI and day 4 post MI were determined and cholesterol ratios (total cholesterol/HDL cholesterol and LDL cholesterol/ HDL cholesterol) were calculated.

Results: On day 1 post MI, mean serum levels of total cholesterol and triglycerides are 190.10 \pm 25.75 and124.98+38.35 respectively. On day 4 posts MI, mean serum levels of total cholesterol and triglycerides are $172.50 \pm 27.05$ and $43.22 \pm 35.64$ respectively. Mean serum total cholesterol levels decreased and mean serum triglyceride levels increased.However cholesterol ratios remain unchanged. On day 1 post MI, total cholesterol /HDL cholesterol ratio and LDL cholesterol/HDL cholesterol ratio were $4.30 \pm 0.36$ and $2.71 \pm 0.40$ respectively. On day 4 post $M I$, total cholesterol $/ H D L$ cholesterol ratio and LDL cholesterol/HDL cholesterol ratio were $4.38 \pm 0.36$ and $2.69 \pm 0.38$ respectively ( no significant change).

Conclusion: Acute myocardial infarction significantly reduces the total serum cholesterol levels and increases the serum triglyceride levels. But the acute myocardial infarction has no significant effect on the cholesterol ratios (LDL cholesterol / HDL cholesterol ratios and cholesterol / HDL cholesterol ratio). So after 24 hours of acute myocardial infarction assessment of cholesterol ratios will be more appropriate than assessing total cholesterol levels.
\end{abstract}

\section{Introduction}

Myocardial infarction generally occurs with the abrupt decrease in coronary blood flow that follows a thrombotic occlusion of a coronary artery previously narrowed by atherosclerosis ${ }^{7}$.

The injury is facilitated by factors such as cigarette smoking, hypertension, dyslipidemia, diabetes and a number of other factors. 
Serum markers that are used for cholesterol risk assessment and management are total cholesterol, low density lipoprotein (LDL) cholesterol level and high density lipoprotein (HDL) cholesterol level. The studies have demonstrated that acute myocardial infarction results in a transient decline in the serum cholesterol levels, which becomes apparent after 24 hours of onset of myocardial infarction and may last for 2 to 3 months. Therefore in situations in which plasma lipid levels are not detemined within 24 hours of the onset of myocardial infarction symptoms, the cholesterol measurements are usually deferred until the effect of acute infarction is fully resolved which may result in an inappropriate delay in the management of hyper cholesterolemia ${ }^{8}$.

\section{Aims and Objectives}

To study the effect of acute myocardial infarction on absolute cholesterol, triglyceride levels and on the ratios of total cholesterol to HDL (High Density Lipoprotein) cholesterol and of LDL (Low Density Lipoprotein) cholesterol to HDL (High Density Lipoprotein) cholesterol.

\section{Materials and Methods}

This is a cross sectional study conducted at the intensive care unit and medical wards of King George hospital, Visakhapatnam from January 2020 to august 2020. This study included patients diagnosed with acute myocardial infarction and diagnosis was made if patients had ischemic type chest pain for $\geq 30$ minutes with evidence of ST segment elevation of $\geq 1 \mathrm{~mm}$ in two anatomically contiguous leads on the ECG or the appearance of a new left bundle branch block. A total of 100 patients were included in the study

Besides clinically examination and routine investigation, the serum lipid profile was measured within the first 24 hours of the onset of symptoms of myocardial infarction and again at day 4 post myocardial infarction.

Following investigations were done for all the patients:

$$
\text { 1. Complete hemogram. }
$$

2. Renal function tests.

3. Serum electrolytes

4. Lipid profile:-

- Total Cholesterol

- LDL cholesterol

- HDL cholesterol

- Triglyceride

- Cholesterol / HDL ratio

- LDL / HDL ratio

5. Electrocardiograph 12 Lead

6. Blood Glucose

7. Echocardiography

\section{Inclusion Criteria}

1) All patients $>18$ years with confirmed diagnosis of acute myocardial infraction

2) Patients who gave informed consent

\section{Exclusion Criteria}

Following patients were excluded from the study

1) Hospital stays of $<4$ days.

2) Already receiving lipid - lowering medications.

\section{Statistical Methods}

Continuous variables were expressed as the mean \pm standard deviation (SD) and the categoric variables were expressed as a percentage. The student's ' $t$ ' test was used to compare lipid values and ratios between day 1 post $\mathrm{M}$ I and day 4 post $\mathrm{M}$ I. A two tailed ' $\mathrm{P}$ ' value of $<0.05$ was considered to be significant

\section{Results}

All serum lipid levels changed significantly between day 1 post myocardial infarction (within 24 hours) and day 4 post myocardial infarction. On day 1 post myocardial infarction the mean total cholesterol value is 190.10 . On day 4 post myocardial infarction, the mean total cholesterol value is 172.50 . On day 1 of post - myocardial infarction, the mean triglyceride value was 124.98. On day 4 of post - myocardial infarction, the mean triglyceride value was 143.22. 
Regarding the Cholesterol ratios, the ratio at total cholesterol to High Density Lipo protein cholesterol (Cholesterol / HDL ratio) on day -1 post myocardial infarction and day 4 post myocardial infarction were 4.300 and 4.380 respectively. The ratio at low density lipoprotein cholesterol to high density lipoprotein cholesterol (LDL/HDL ratios) on day -1 post myocardial infarction and day- 4 post myocardial infarction were 2.710 and 2.690 respectively.

COMPARISON OF THE SERUM LIPID VALUES AND RATIOS BETWEEN WITHIN 24 HOURS OF MYOCARDIAL INFARCTION AND DAY-4 POST MYOCARDIAL INFARCTION

\begin{tabular}{|l|c|c|c|}
\hline \multicolumn{1}{|c|}{ Serum lipids } & $\begin{array}{r}\text { Within 24hrs of } \\
\text { Myocardial infarction }\end{array}$ & $\begin{array}{c}\text { Day-4 post } \\
\text { Myocardial } \\
\text { infarction }\end{array}$ & $\begin{array}{c}\text { ' } \mathbf{Z} \text { ' and 'P' } \\
\text { Values }\end{array}$ \\
\hline $\begin{array}{l}\text { Total cholesterol } \\
(\mathrm{mg} / \mathrm{dl})\end{array}$ & $190.10 \pm 25.75$ & $172.50 \pm 27.05$ & 6.33 \\
\hline Triglycerides (mg/dl) & $124.98 \pm 38.35$ & $143.22 \pm 35.64$ & 4.02 \\
\hline Total cholesterol & $4.30 \pm 0.36$ & $4.38 \pm 0.36$ & 0.33 \\
\hline HDL cholesterol ratio & & & \\
\hline LDL cholesterol & $2.71 \pm 0.40$ & $2.69 \pm 0.38$ & 0.06 \\
\hline HDL cholesterol ratio & & & \\
\hline
\end{tabular}

\section{Discussion}

Acute myocardial infarction results in a significant decrease in the serum levels of total cholesterol, LDL cholesterol and HDL cholesterol ${ }^{2}$. The post myocardial infarction decline in serum cholesterol occurs because of the acute - phase response, and is of greatest extent by days 4 to day 5 post myocardial infarction.By day 4 to 5 post myocardial infarction, there is a significant decrease in the serum concentrations of apoprotein A-1 and apoprotein-B reflecting the maximum decrease in the serum cholesterol level by the time $^{1}$.

This paradoxical rise in serum triglycerides is due to increase in serum $-\mathrm{C}$ reactive protein level which may increase to levels that are several hundred - fold higher than baseline 4 days after and myocardial infarction ${ }^{5}$. in patients whose cholesterol levels were not determined with in the first 24 hours after the onset of acute infarction can lead to a delay in initiating the appropriate cholesterol lowering therapy for the secondary prevention of future coronary events.

Several large - scale epidemiologic studies have shown that the total cholesterol / HDL cholesterol ratio and LDL cholesterol/HDL cholesterol ratio are also strong predictors of coronary artery disease events ${ }^{6}$.in certain situations in which the plasma cholesterol levels are not measured within the first 24 hours after the onset of acute myocardial infarction, cholesterol ratios determined from the serum cholesterol measurements taken after 24 hours of the onset of acute infarction could be used reliably for cholesterol risk assessment ,because cholesterol ratios didn't change significantly.

\section{Conclusion}

Following acute myocardial infarction the total serum cholesterol level falls significantly and the triglyceride level rises significantly. Therefore measurement of absolute levels of serum cholesterol and triglycerides following acute myocardial infarction are not valid in risk assessment 24 hours after infarction. But acute myocardial infarction does not affect the cholesterol ratios. (Cholesterol / HDL and LDL / HDL cholesterol ratios) even 24 hours after infarction. Therefore following acute myocardial infarction, the cholesterol ratios are valid and very useful in risk assessment.

\section{Limitation}

This study is done in a limited number of patients. Results may vary when done in large number of patients.

\section{References}

1. SMIth SC Jr, Greenland P, Grundy SM. Identifying the high risk patient for primary prevention; executive summary. Circulation 2000; $101 ; 111-116$. 
2. Avogar OP, Bon GB, Cazzolato $G$ et al. Variations in apolipoproteins B and A-1 during the course of myocardial infarction Eur J Clin Invest $2008 ; 8 ; 121$ - 129.

3. Rosenson RS. Myocardial injury: the acute phase response and lipoprotein metabolism. J Amcollcardial 2003; 22 ; $933-940$.

4. Pyfe T, Baxter RH, Cochran DM, et al. Plasma lipid changes after myocardial infarction, Lancet $2001 ; 2$ : $997-1001$.

5. Jackson R, Scragg R, Marshall R, et al. Changes in serum lipid concentrations during first 24 hours after myocardial infarction. BMJ 1997 ; 294 : 1588 - 1589.

6. Heldenberg D, Rubinstein A, Levtor O, et al Serum lipids and lipoprotein concentrations during the acute phase of myocardial infarction. Atherosclerosis $2000 ; 35: 433-437$

7. Castelli WP. Epide MIology of coronary heart disease; the FraMIngham study. Am J Med $2004: 27: 4-12$.

8. Ryder RE, Hayes TM, Mulligan IP, et al. How soon after myocardial infarction should plasma lipid values be assessed? BMJ $2004 ; 289 ; 1651$ - 1653. 